\title{
High-performance adaptive hybrid wireless NoC architecture based on improved congestion measurement
}

\author{
Jianhua $\mathrm{Li}^{1,2 a)}$, Ning $\mathrm{Wu}^{1}$, Yongliang $\mathrm{Hu}^{2}$, and Xiaoqiang Zhang ${ }^{1}$ \\ ${ }^{1}$ College of Electronic and Information Engineering, \\ Nanjing University of Aeronautics and Astronautics, 210016, China \\ ${ }^{2}$ Institute of Computer Application, Taizhou University, 317000, China \\ a)ljh2007@tzc.edu.cn
}

\begin{abstract}
In view of the high energy consumption and latency problem due to multi-hop wired links between distant cores of traditional large-scale Network-on-Chip, a virtual torus-based adaptive wireless NoC architecture has been proposed when analyzing the existing several wireless NoC architectures. By not only adopting the automatic detection and dynamic bandwidth allocation mechanism to hot wireless link based on the sensing parameter for improved congestion measurement, but also designing the Dynamic Allocation Control Circuit Module (DACCM) for transmitter, the intra-chip topology and link bandwidth could be adaptively adjusted as different traffic patterns. Experimental results show that the proposed architecture has more significant latency improvement and energy saving under different traffic patterns or real application such benchmark as FFT.
\end{abstract}

Keywords: Wireless Network-on-Chip (WiNoC), adaptive topology, congestion measurement, dynamic bandwidth allocation

Classification: Integrated circuits

\section{References}

[1] W.-H. Hu, C. Wang and N. Bagherzadeh: Euromicro International Conference on Parallel, Distributed and Network-based Processing, February (2012) 483. DOI:10.1109/PDP.2012.19

[2] ITRS, http://www.itrs.net/Links/2011ITRS/Home2011.htm.

[3] M. Bialkowski and A. Abbosh: IET Microw. Antennas Propag. 4 (2010) 1665. DOI:10.1049/iet-map.2009.0238

[4] S. F. Mahmoud and A. R. AlAjmi: IEEE Trans. Nanotechnol. 11 (2012) 640. DOI:10.1109/TNANO.2012.2190752

[5] D. Zhao and Y. Wang: IEEE Trans. Comput. 57 (2008) 1230. DOI:10.1109/TC. 2008.86

[6] S. Yin, Y. Hu, Z. Zhang, L. Liu and S. Wei: IEICE Trans. Electron. E95-C (2012) 495. DOI:10.1587/transele.E95.C.495

[7] W. Chifeng, H. Wen-Hsiang and N. Bagherzadeh: Proc. 19th Euromicro International Conf. Parallel, Distributed and Network-Based Processing (PDP) 
(2011) 409. DOI:10.1109/PDP.2011.37

[8] A. Ganguly, K. Chang, S. Deb, P. P. Pande, B. Belzer and C. Teuscher: IEEE Trans. Comput. 60 (2011) 1485. DOI:10.1109/TC.2010.176

[9] L. Wang, Z. Guo, P. Lv and Y. Jiang: IJACSA 5 (2014) 168. DOI:10.14569/ IJACSA.2014.050424

[10] D. DiTomaso, A. Kodi, D. Matolak, S. Kaya, S. Laha and W. Rayess: IEEE/ ACM International Symposium on Networks on Chip (NoCS) (2013) 1. DOI: $10.1109 / \mathrm{NoCS} .2013 .6558400$

[11] S.-B. Lee, S. Tam, L. Pefkianakis, S. Lu, M. F. Chang, C. Guo, G. Reinman, C. Peng, M. Naik, L. Zhang and J. Cong: ACM Mobicom (2009) 217. DOI:10. $1145 / 1614320.1614345$

[12] J. Li, N. Wu and Y. Hu: Sens. Transducers J. 155 [8] (2013) 86.

[13] A. B. Kahng, B. Li, L.-S. Peh and K. Samadi: IEEE Trans. Very Large Scale Integr. (VLSI) Syst. 20 (2012) 191. DOI:10.1109/TVLSI.2010.2091686

[14] S. Deb, K. Chang, X. Yu, S. P. Sah, M. Cosic, A. Ganguly, P. P. Pande, B. Belzer and D. Heo: IEEE Trans. Comput. 62 (2013) 2382. DOI:10.1109/TC. 2012.224

\section{Introduction}

Network-on-Chip (NoC) has emerged as an interconnection and communication architecture for complex Systems-on-Chip (SoC). Despite its many advantages, the drawbacks on high latency and power consumption, however, seriously hinder the further performance enhancement of multicore $\mathrm{SoC}$ with the increasing complexity [1]. On the one hand, nice topologies (e.g. Torus) have suffered from limitations in long wiring over the whole network and high power consumption. According to the International Technology Roadmap for Semiconductors (ITRS) [2], the contribution of metallic interconnection to chip power consumption increase from $51 \%$ in the 0.13 um technology up to $80 \%$. On the other hand, the data exchange between the distant IP cores causes more latency and power consumption arising from multihop communication. It's more clear that the performance on conventional NoC bottlenecks confines the performance improvement of IC with the expansion of network size.

More recently, the Wireless NoC (WiNoC) has been proposed as a promising solution by integrating miniaturized on-chip antennas and transceivers to enable intra-chip wireless interconnection and communication [1,3,4], wherein the multihop wired links have been replaced by single-hop long-range wireless links to solve the wiring and performance limitations radically. Although the WiNoC architecture using all wireless links with UWB technology has been explored to transfer data between different nodes [5], excessive wireless nodes cause high power consumption and area overhead. To design a dedicated NoC architecture, the heuristic algorithm following theory of Network Calculus has been adopted to allocate wireless link statically for hybrid wired/wireless NoC [6].

In [7], performance of WiNoC architecture based on conventional wired 2D Mesh NoC architecture called NePA has been shown to improve by a hybrid mechanism taking advantage of both wired and wireless communications (denoted as NePA-WiNoC). The every subnet will be greater and easier to become congested 
with the network scale expansion. Ganguly et al. proposed a hybrid architecture using several centralized wireless hubs connected following principles of smallworld graphs (denoted as SW-WiNoC) [8]. Although single-hop communication could improves the network performance, the centralized hub that has been connected to many IP cores directly, causes high area overhead and congestion in convergent port of hub. After taking into account the number of wireless nodes, wireless links and their even distribution, the 2-Level Hybrid Mesh architecture (denoted as 2LHM-WiNoC) with a layered architecture consisted of lower-layer wired and upper-layer multihop wireless mesh topology has been shown to avoid network congestion [9].

Respecting the above-mentioned various WiNoC topologies, static channel allocation has been adopted to improve performance of the traditional metallic NoC, which means that no matter whether and how much data is ready to send, the fixed bandwidth will be allocated to the wireless links and therefore the channel bandwidth may be wasted on them. Moreover, the intra-chip topology of upperlayer wireless network is unable to adjust self-adaptively according to traffic patterns. Especially, how to adaptively adjust the intra-chip topology and link bandwidth is critical to improving performance of WiNoC under the limited channel number and total bandwidth.

An adaptive wireless $\mathrm{NoC}$ architecture (A-WiNoC), which uses adaptive and energy efficient wireless transceivers, has been proposed to improve network power and throughput by adapting algorithm taking link utilization statistics [10]. In spite of significant performance gains, the A-WiNoC architecture is not easy to avoid the growing congestion in the router connected by increasing number of cores.

Therefore, in this work, we propose an Adaptive Hybrid Wireless NoC architecture based on the Virtual Torus interconnection (VT-AWiNoC) by taking into full account the inherent adaptability and the factors such as the channel number, channel capacity and congestion of wireless links. The adaptive transmitter and channel are allocated by the Dynamic Allocation Control Circuit Module (DACCM) to the most congested hotspot in wireless links following the improved method of congestion measurement, and thus the intra-chip topology can be changed adaptively according to traffic patterns.

\section{The proposed adaptive hybrid wireless NoC topology}

Topology design is the research foundation of wireless NoC similar to the wired NoC with pure metallic interconnection. Not only the layout and interconnection of wired nodes but also the number and placement of wireless links will have significant impacts to the performance of hybrid wireless NoC. Due to the limitation in the number of available channel, thus the limited number of wireless channels should be utilized fully to improve the communication capacity of WiNoC while we work on topology design. An adaptive hybrid WiNoC architecture (named VT-AWiNoC) with high performances of lower latency and power consumption has been proposed by analyzing the shortcomings of existing topology in WiNoC. The proposed architecture is shown in Fig. 1. 
We suppose that there is an existing wired 2D-Mesh NoC with scale of $\mathrm{n} \times \mathrm{n}$ ( $n=18$ as shown in Fig. 1), where $n$ is an integer greater than 6 since the wireless NoC is usually used to solve the multihop communication and wiring problem of large scale network. The construct rules of proposed adaptive topology are shown as follows:

(1) Firstly, we divide the original $\mathrm{n} \times \mathrm{n} 2 \mathrm{D}$-Mesh $\mathrm{NoC}$ into several clusters of $6 \times 6$, and then divide every cluster into several blocks of $3 \times 3$. As shown in Fig. 1, there are nine clusters (from Cluster0 to Cluster8) in whole network, four blocks per cluster (from Block0 to Block3).

(2) The center router in each block is replaced by a wireless node consisting of a wireless router (WR) and block transmitter. The wireless node connects with other neighbor wireless nodes locating only in a horizontal or vertical direction using static wireless links. Individual routers are marked as $\mathrm{R}(\mathrm{cn}, \mathrm{bn}, \mathrm{zn})$ where $\mathrm{zn}$, bn and $\mathrm{cn}$ represent the router number in per block, block number in per cluster and cluster number, respectively.

(3) We define the Opposite Cluster (OC) as the farthest clusters locating only in a horizontal or vertical direction with itself. In the same way, define the Opposite WR (or Opposite Blocks, OB) as the farthest WRs (or OB) locating only in a horizontal or vertical direction with itself. As shown in Fig. 1, the Cluster0's OC are Cluster2 in the horizontal direction and Cluster6 in the vertical direction. Similarly, the WR0's Opposite WR are WR9 and WR26 in the horizontal and vertical direction, respectively. The WR1's Opposite WR, however, which is not existing in the horizontal direction, is only WR27 in the vertical direction.

(4) Additionally, an adaptive Cluster Transmitter (CT) placed in the center of each cluster has been connected to $\operatorname{Max}(b n)+1$ WRs via wired links. Where $\operatorname{Max}(b n)$ represents the maximum of variable bn such as bn $=3$ shown in Fig. 1. The $\operatorname{Max}(b n)+1$ WRs share the CT in cluster, meanwhile, we insert an adaptive wireless link between neighbor CTs or Opposite CTs in the horizontal or vertical direction, where denote an adaptive wireless links inserted between Opposite CTs as Long Wireless Link (LWL). The only one WR monopolize the CT if cluster number equals to one.
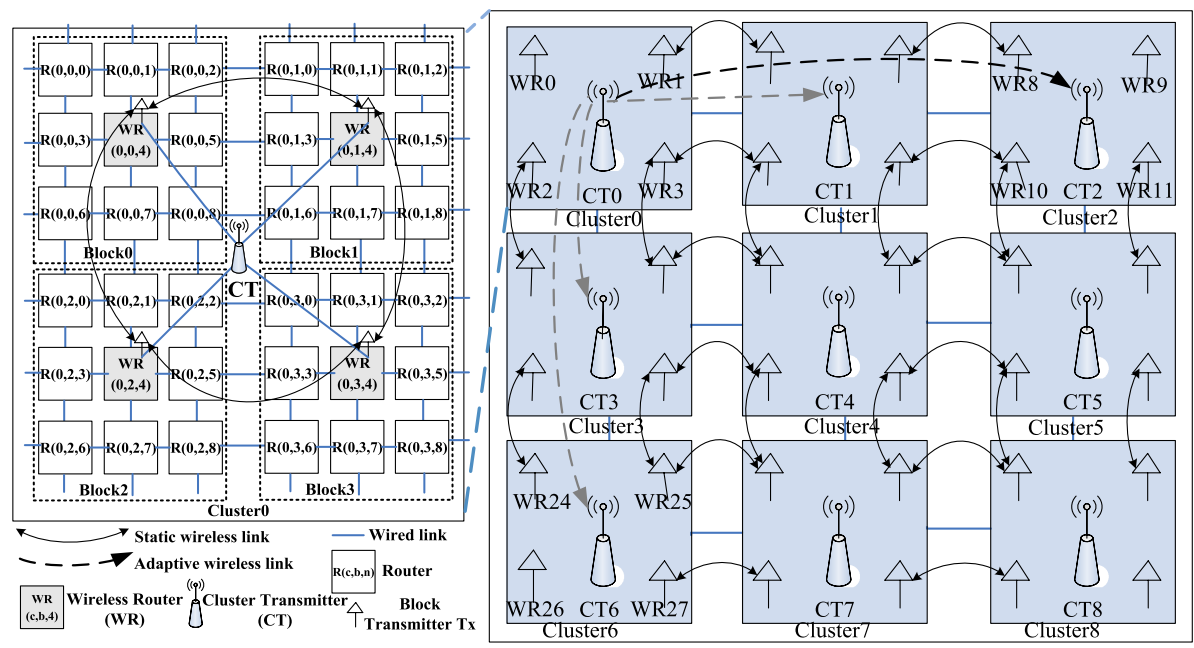

Fig. 1. Proposed VT-AWiNoC architecture. 
(5) To reduce the number of hops and avoid the local congestion in CT due to the heavy traffic load, we limit that the shared CT is only used to communicate between neighbor WRs of neighbor clusters or opposite WRs, not between neighbor WRs in some cluster. Therefore, as shown in Fig. 1, the CT0 will be dynamically allocated to the eight wireless links, such as between WR0 and WR9 (LWL), between WR0 and WR26 (LWL), between WR1 and WR4, between WR1 and WR27 (LWL), between WR2 and WR12, between WR2 and WR11 (LWL), between WR3 and WR6 or between WR3 and WR13. The allocation mechanism based on congestion measurement has been discussed in subsequent section.

According to the description above, the upper-layer wireless will form a Mesh network while all the CTs aren't used for LWL. Similarly, the upper-layer static wireless links and adaptive wireless links form a virtual Torus network if all the edge CTs are used to communicate for LWL. If the size of some divided $6 * 6$ cluster or $3 * 3$ block is inadequate (i.e. the $\mathrm{n}$ is not multiple of 6 ), we see it as a complete cluster or block instead of adding redundant nodes to avoid the additional power consumption and area overhead in the case of without affecting the design of adaptive topology.

The structure of WR and its interconnecting with $\mathrm{CT}$ as shown in Fig. 2. Each WR includes five input/output ports (E, S, W, N and L), four wireless input/ output ports (BW_E, BW_S, BW_W, BS_N, on each for east, west, north and south WR), Routing Arbitrator (RA) and Local Congestion Measure Unit (LCMU) with arbitration and traffic counter. The LCMU collects statistical congestion information on each link of WR and transmits the congestion measurement data to Global Congestion Measure Unit (GCMU) with comparator, and then GCMU by using port 1 to $\operatorname{Max}(\mathrm{bn})$ obtains the output data from LCMUs of other WRs.

Each wireless output port has been connected to a different static transmitter (Tx) except the edge ports from edge WRs. As shown in Fig. 2, only the two nonedge ports (BW_S_out and BW_E_out) of WR0 have connect a Tx respectively, however the four output ports of WR3 all connect a Tx, respectively.

The Tx, which works on a unique frequency (from f1 to f4) by sub-channel division based on FDMA technique according to literature [8], has been connected statically to the adjacent WRs locating in horizontal or vertical direction. According to the above construct rules of adaptive topology, only two of all the wireless output ports of WR locating different position have been simultaneously connected to the CT in some cluster. As shown in Fig. 2, only the two ports (i.e. BW_N_out and BW_W_out) of WR0 have been connected to the CT0 simultaneously, that's to say, BW_N_out and BW_W_out have all been connected to CT0 for long-distance communication with WR26 and WR9 respectively. Similarly, only the two ports (i.e. BW_S_out and BW_E_out) of WR3 have been connected to CT0 simultaneously. The intra-chip topology of upper-layer wireless network can be adaptively adjusted according to different traffic patterns if the CTs have been dynamically allocated to different WRs. 


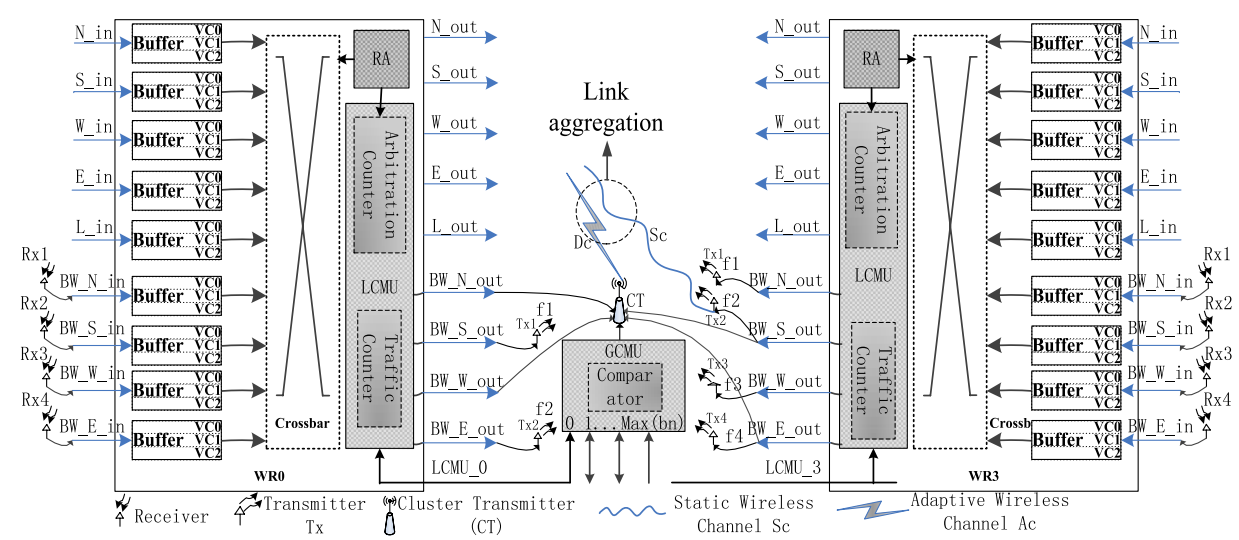

Fig. 2. The structure of WR and its interconnecting with CT.

\section{Improved congestion measurement and dynamic bandwidth allocation mechanism of wireless links}

It is possible to switch a CMOS transistor as fast as $500 \mathrm{GHz}$ at $32 \mathrm{~nm}$ CMOS, in addition, the total aggregate data rate that can be as high as $320 \mathrm{Gbps}$ is enough to the high-bandwidth demand of WiNoC [11]. But how to dynamically allocate the idle channels to high-traffic links for solving of congestion problem is the critical factor for performance improvements of WiNoC.

The congestion level of link is usually predicted by the link bandwidth utilization [10], but more likely link is congested due to the contention for a same output port arising from many input ports in NoC. The simulation demonstrates that the link utilization shows just the past practical traffic condition of output port in a selected time window $\mathrm{W}$, but not the future traffic arising from the input ports waiting for the same output port. The bandwidth requirement and congestion at next time are more dependent on the communication traffic waiting for passing through output port. Therefore, the link bandwidth requirement at the next $\mathrm{W}$ relates with the contention at the last cycle of previous W. According the above communication characteristics of WiNoC, we define the Congestion Measurement (CM) of wireless link between two adjacent wireless nodes as follows:

$$
C M_{a(j) b(i)}=\frac{\mathrm{Q}_{(|W|-1), a(j)}}{|W|} \sum_{t=0}^{(|W|-1)} Q_{t, a(j)} \cdot r_{a(j) b(i)}
$$

Where $r_{a(j) b(i)}$, which is proportional to traffic $\mathrm{C}$ at the time window $\mathrm{W}$ and inversely proportional to theoretical bandwidth $\mathrm{B}$, represents the bandwidth utilization of wireless link $l_{a(j) b(i)}$ between adjacent wireless router a and wireless router $\mathrm{b}$ and shows the past communication traffic and congestion, is defined as:

$$
r_{a(j) b(i)}=\frac{C_{a(j) b(i)}}{|W| \cdot B_{a(j) b(i)}}
$$

Where $\mathrm{Q}_{(|W|-1), a(j)}$ is the traffic waiting for passing through the output port $\mathrm{j}$ at the last time $t=|W|-1$ of time window. $Q_{t, a(j)}$, which is the total traffic of all input ports waiting for passing through output port $\mathrm{j}$ of router at time $t$, is defined as:

$$
Q_{t, a(j)}=\sum_{k=0}^{|I|+|U|-1} q_{t, a(k) a(j)} \cdot g_{t, a(k) a(j)} \quad k \neq j
$$


Where the sum of $|I|$ and $|U|$ equals to nine for the $9 \times 9 \mathrm{WR}, q_{t, a(k) a(j)}$ represents the traffic of input port $\mathrm{k}$ waiting for passing through output port $\mathrm{j}$ at time $\mathrm{t}, g_{t, a(k) a(j)}$ represents whether the input port $\mathrm{k}$ is waiting for output port $\mathrm{j}$, is defined as:

$$
g_{t, a(k) a(j)}= \begin{cases}1 & \text { yes } \\ 0 & \text { no }\end{cases}
$$

The parameter CM embodies the priority service for the more congested link and allocation on demand based on traffic analysis, and if its value is bigger, the congestion level of link $l_{a(j) b(i)}$ is higher.

To reduce the circuitry complexity, the bit stream of output link could be counted by the hardware traffic counter instead of the value of $C_{a(j) b(i)}$. Additionally, when the number of flits waiting to pass through router and flit width in direct proportion to traffic $q_{t, a(k) a(j)}$ of output port k could be counted or is the know value, $Q_{t, a(j)}$ can be considered as the number of input port waiting for passing through the output port $\mathrm{j}$. Thus we can use the hardware arbiter counter to count the number of input ports waiting for transmission in the stage of routing arbiter simultaneously and further simplify the circuitry. It can be seen that we just equip with the additional hardware arbiter and traffic counter as shown in Fig. 2 after by simplifying the circuitry for computing the value of CM in WR. Thus the hardware overhead is negligible to the WR.

The above improved CM model is validated using simulation by selecting randomly a wireless link from network. Such as for the wireless link between output port BW_E_out at WR1 and port BW_W_in at WR4, the changing curve of normalized $\mathrm{CM}$ with time points for three different parameter models at $|W|=100$ as shown in Fig. 3. It can be seen that the normalized CM of improved CM model is more close to the measured value of congestion level and reduces the average error of $33.89 \%$ compared with CM model based on bandwidth utilization.

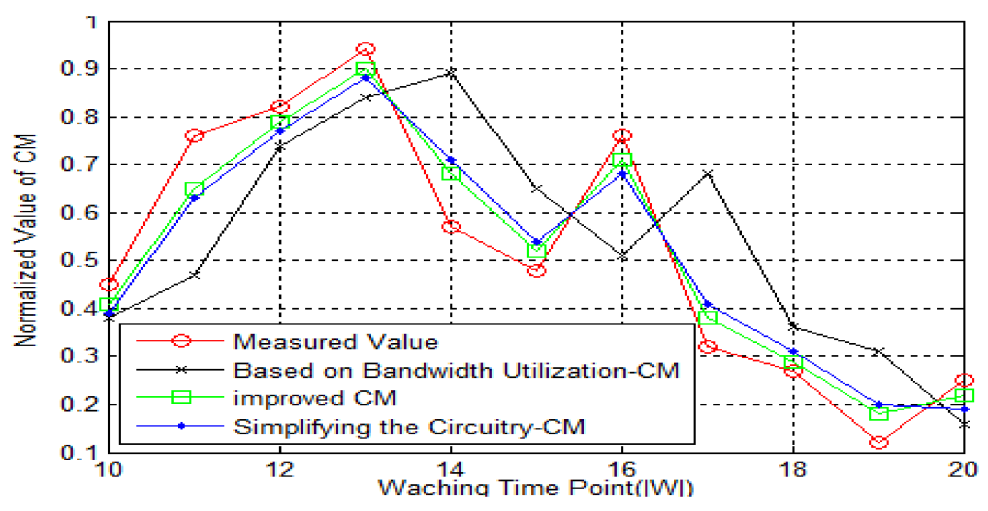

Fig. 3. The normalized CM under different parameter model. 
frequency. As shown in the Fig. 4(a), the proposed VT-AWiNoC architecture needs only 8 static channels $(\mathrm{Sc} 0-\mathrm{Sc} 7)$ and 4 adaptive channels (Ac0-Ac3). The $\mathrm{n} \times \mathrm{n}$ VTAWiNoC architecture needs $\mathrm{m}^{2}$ (where $m=n / 6$ ) adaptive cluster transmitters (CTs) totally. In this architecture, the wireless link between every two adjacent WRs has been allocated only one static channel (Sc) connecting the network topology at all times. To reduce the unnecessary complexity of the network, each CT has been allocated only one Ac. As shown in Fig. 4(b), there are four Acs (Ac0Ac3) which are allocated dynamically for data transmission according to the traffic pattern, and thus it offers the additional bandwidth to some hot wireless links.

Since the CT could only be used to communicate between adjacent or Opposite WRs, the Ac of CT is reused dynamically by the 8 wireless links locating in 4 WRs of block. Assignment of Ac0 from CT0 is shown as red dotted arrows and Ac2 from CT2 is shown as green dotted arrows in Fig. 4(b).
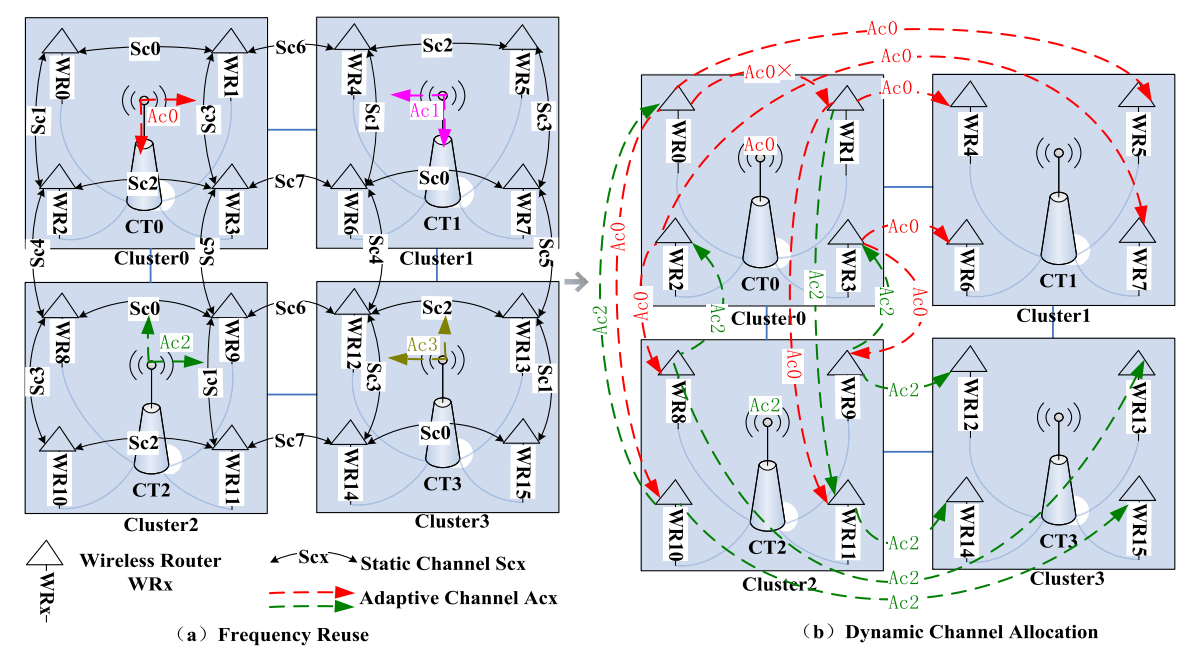

Fig. 4. Frequency reuse and mechanism of dynamic channel allocation.

The DACCM, which used for dynamic allocating the CT, is the critical component for the adaptive adjustment of intra-chip topology and link bandwidth as shown in Fig. 5.

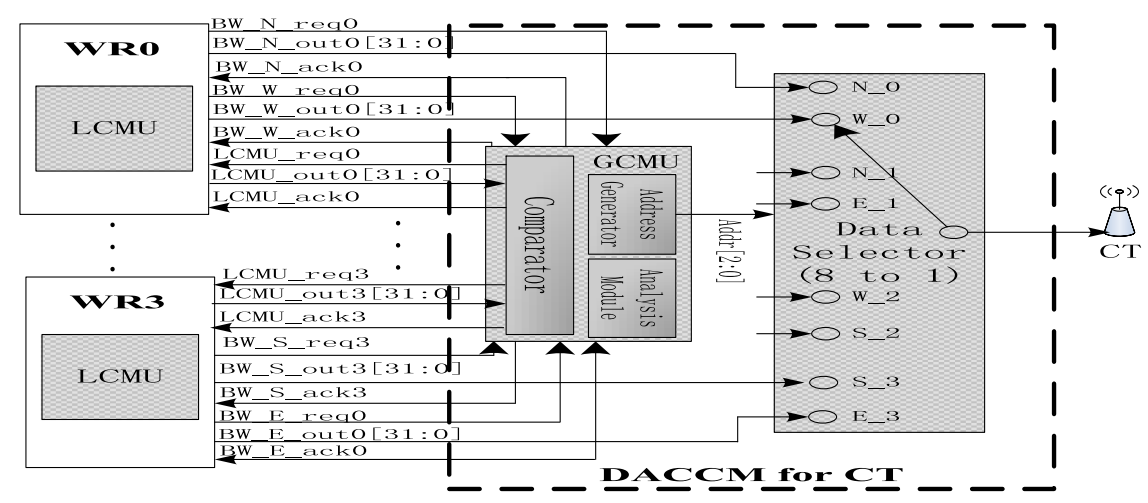

Fig. 5. The DACCM for transmitter CT. 
According to the construct rules of proposed adaptive topology, the work process of DACCM for dynamic allocating transceiver shown in Fig. 5 as follows:

(1) Setting the time window W, each LCMU analyzes the communication traffic and link utilization within window W.

(2) GCMU sends requesting information about data of link congestion measurement to every LCMU in cluster.

(3) At the end of time window W, each LCMU computes the value of congestion measurement on only two of all the wireless output links of local WR, and then sends the CM value as a packet to GCMU.

(4) GCMU analyzes the CM packets received from output links of four WRs. Whatever the value of CM, it will gives more priority that allocate the Ac to the LWL. If there are many LWLs, the Ac will be preferentially allocated to the LWL with maximum CM. Otherwise, without LWL, it will allocate the Ac to the static wireless link with maximum CM. The wireless links with static and dynamic channels have eventually been merged into a logical Link by using link aggregation. Using the dynamic switching of CT between WRs in cluster by data selector, it will adjust the intra-chip topology while increasing bandwidth.

(5) The LWL that completes the data communication in W will give up the Ac and then the Ac will be allocated to the another wireless link until the end of W.

\section{Communication mechanism of VT-AWiNoC}

For the proposed VT-AWiNoC, packets traverse the lower-layer wired 2D Mesh network on a deadlock-free path following deterministic XY dimension order routing. The routing algorithm in the upper-layer ring with virtual torus using static and adaptive wireless links can be the TXY routing with two virtual channels to avoid deadlocks. Additionally, the upper-layer wireless links and lower-layer wired links could also form the circular dependency, so each input port has three virtual channels (VC0-VC2) used to avoid deadlocks as shown in Fig. 2.

As the wormhole routing is adopted, the computation is done only once for the header flit, and the rest of the flits follow the header to traverse through the wired/ wireless router until they reach their destinations. When transferring flits between WRs in VT-AWiNoC, a packet may be transmitted through the wired links, the wireless links, or a mix of the two. For a packet whose source node and destination node are located in the same block, there's no need to use the wireless links, thus the packet will be sent from source to destination by wired links using XY routing. For a packet whose source node and destination node are from different blocks, the factor $\delta$ for congestion control is set to avoid excessive use of wireless links and produce hotspot [7]. The routing decision algorithm combing XY with TXY is adopted in our proposed architecture to avoid deadlocks.

\section{Performance evaluation}

To the different characteristics of two types of antennas, the modeled intra-chip wireless propagation channels based on the 3-D Descartes coordinate system and the ray tracing for hybrid WiNoC is used in this paper [12]. The power consumption for wired links including dynamic power and leakage power for $5 \times 5$ router 
and metallic wires was obtained from Orion 2.0 power simulator [13]. For the $20 \mathrm{~mm} \times 20 \mathrm{~mm}$ die, supposing the high resistivity silicon substrate $(\rho=5 \mathrm{k} \Omega-\mathrm{cm})$ and oxide layer (e.g., AlN) of thickness $633 \mu \mathrm{m}$ are used in hybrid WiNoC, the loss between antennas $\mathrm{PL}_{\mathrm{G}}$ is about $32 \mathrm{~dB}$ for $20 \mathrm{~mm}$ communication distance (about $9 \mathrm{~dB}$ at $1 \mathrm{~mm}$ distance) according to literature [12]. Since the power consumption of transceiver is found to be $36.7 \mathrm{~mW}$ at $16 \mathrm{Gbps}$ for WiNoC, and thus the corresponding power consumption is $2.3 \mathrm{pJ} /$ bit [14].

To evaluate the additional power consumption and area of the circuits realizing the adaptive adjustment of topology, the DACCA used for allocating transceiver CT dynamically as shown in Fig. 5 has been synthesized using Synopsys Design Compiler and $90 \mathrm{~nm}$ CMOS technology as shown in Table I, below.

Table I. The power consumption and area of DACCM

\begin{tabular}{l|l}
\hline Energy $(\mathrm{pJ} /$ bit $)$ & Area $\left(\mathrm{mm}^{2}\right)$ \\
\hline 0.0116 & 0.0325 \\
\hline
\end{tabular}

Based on the above analysis, we developed the system level simulation platform for four different Hybrid WiNoC topologies (i.e. SW-WiNoC, NePA-WiNoC, 2LHM-WiNoC and VT-AWiNoC) by modifying the cycle accurate simulator Noxim and embedding the models for power consumption and latency. To ensure the fair comparison for performance evaluation, the same network sizes (e.g. 144, 324 and 576 nodes), traffic patterns and real applications were used in our simulation. The wired links and wireless links were driven with clock of frequency $1 \mathrm{GHz}$ and $16 \mathrm{GHz}$, respectively. Here we assume the flit size is 16 bits for a fair comparison with wired NoC. The input and output ports have three virtual channels, each having a buffer depth of 4 flits. Fig. 6 shows the throughput as function of injection load for four hybrid WiNoC with different traffic patterns (e.g. uniform random traffic, 20\% hotspot and real application FFT). As illustrated in Fig. 7, the comparison of latency for different network sizes under 0.2 injection load.

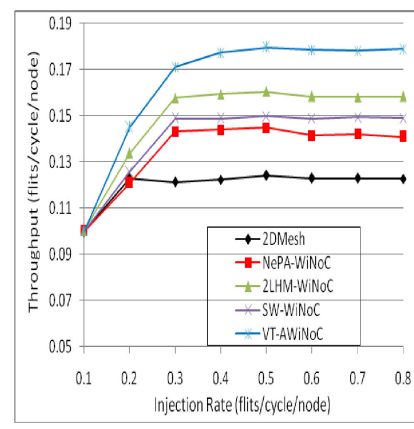

(a) Uniform Random Traffic

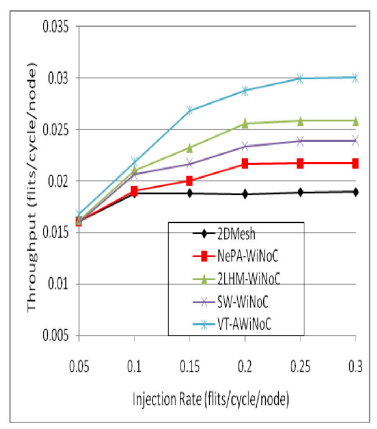

(b) $20 \%$ Hotspot Traffic

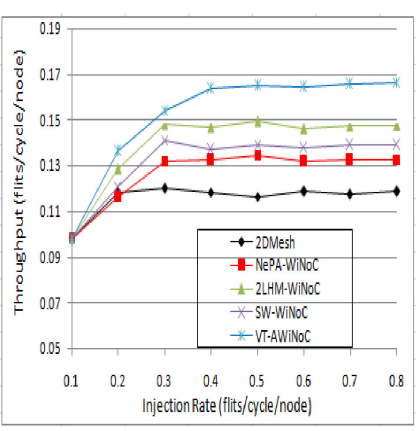

(c) Real Application FFT

Fig. 6. The comparison of throughput under different traffic patterns for five NoC topologies (nodes $=144$ ). 


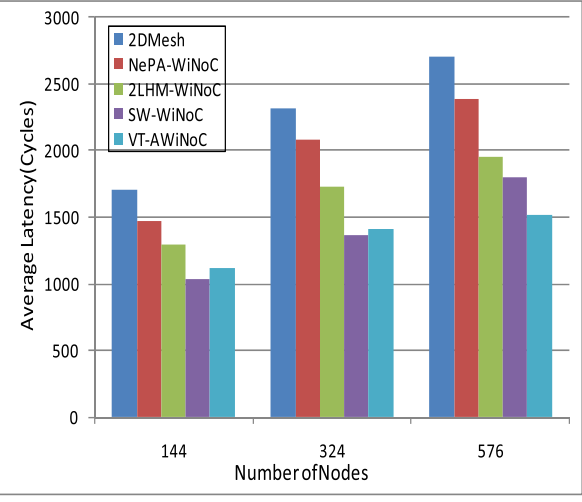

(a) Uniform Random Traffic

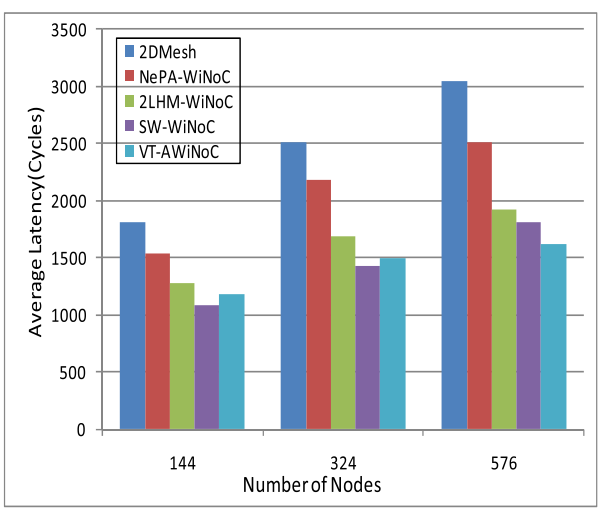

(b) $20 \%$ Hotspot Traffic

Fig. 7. The comparison of average latency for different traffic patterns (Injection Load $=0.2$ ).

The average power consumption per packet under different traffic patterns and real application as shown in Fig. 8. Under the uniform random traffic pattern and $20 \%$ hotspot, the simulated results show that the VT-AWiNoC is still better than the other four topologies on energy consumption.

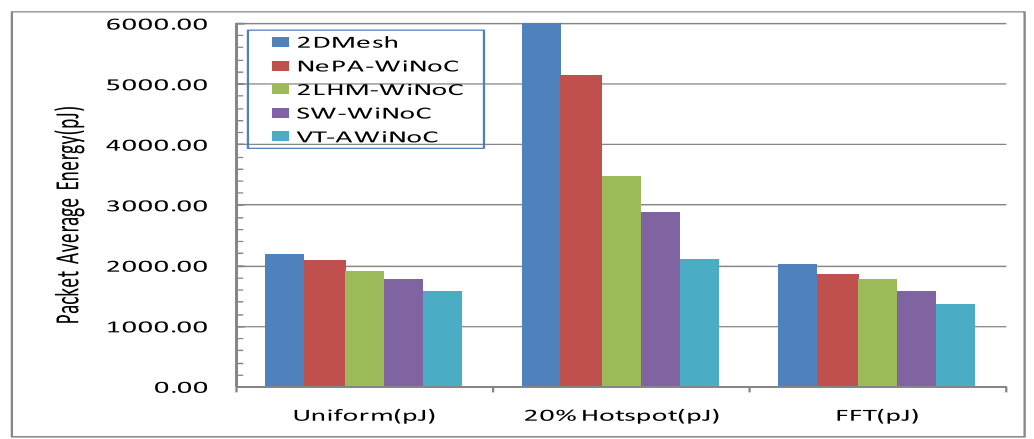

Fig. 8. The average power consumption per packet under different traffic patterns and real application.

The area for $5 \times 5$ router and $1 \mathrm{~mm}$ long metallic link with 32-bit was estimated using Orion 2.0 in $45 \mathrm{~nm}$ technology and found to be $0.0826 \mathrm{~mm}^{2}$ and $0.0052 \mathrm{~mm}^{2}$, respectively. The area of wireless link including the CT consisted of TDM, modulator-demodulator, PA and antennas is about $0.0854 \sim 0.1410 \mathrm{~mm}^{2}[7,8]$. The area of $9 \times 9$ WR is $0.2485 \mathrm{~mm}^{2}$ (where $0.0236 \mathrm{~m}^{2}$ for buffer, $0.2017 \mathrm{~mm}^{2}$ for crossbar). So it can be seen that the wireless links will increase the area in WiNoC, but for the $400 \mathrm{~mm}^{2}$ die, the area overhead of circuits and wireless links for adaptive topology is about $1.04 \% \sim 1.26 \%$. On the one hand, the VT-AWiNoC topology has higher gain in terms of throughput, latency and power consumption at the cost of little area overhead. On the other hand, the proposed topology avoids the area overhead of long metallic wire and wiring limitation.

\section{Conclusions}

In this work, we propose and compare the performance of VT-AWiNoC architecture to other NoC designs including 2D-Mesh and hybrid WiNoC. By designing of 
DACCM and using automatic detection and dynamic bandwidth allocation mechanism to hot wireless link based on improved congestion measurement, we have achieved our aim of adjusting self-adaptively intra-chip topology according to different communication traffic patterns.

The experimental results demonstrate the proposed adaptive hybrid WiNoC architecture gaining high performance is superior to the other hybrid WiNoC topologies or 2D-Mesh NoC due to the dynamic bandwidth allocation mechanism in VT-AWiNoC to hot wireless link based on the sensing parameter for improved congestion measurement. Compared with pure wired 2D-Mesh NoC, the VTAWiNoC avoids plenty of intermediate nodes and metallic wires for packet transmitting by inserting long-range wireless links with lower energy. Especially, the lager the network, the more obvious performance enhancement.

\section{Acknowledgements}

This work is supported by the National Natural Science Foundation of China under Grant No. 61376025, the Natural Science Foundation of Zhejiang Province of China under Grant No. LY13F020012, Science and Technology Project of Jiangsu Province of China under Grant BE2010003. 